\title{
Vibration Analysis of Nonlinear Tapered Functionally Graded Beams Using Collocation Method with Gauss-Legendre Points
}

\author{
Reza Adelkhani ${ }^{1}$ and Jaafar Ghanbari ${ }^{1}$ \\ ${ }^{1}$ Qom University of Technology
}

June 26, 2020

\begin{abstract}
Free transverse vibration of variable cross-section cantilever FG beams with nonlinear profiles is investigated in this paper. Four thickness functions, namely, linear, parabolic, sinusoidal, and exponential functions are assumed for variation of the cross-section of the beam. Linear and exponential grading rules are covered in this paper. The governing differential equation is solved using the weighted residual collocation method with the exact solution shape functions for the uniform beam as the trial functions. This choice for the trial functions showed an increase in the convergence rate. The Gauss-Legendre points are used as the collocation points to reduce the fluctuations in the convergence curve as usually encountered in the point collocation method. The effects of the taper parameter for all kinds of thickness functions on the natural frequencies are studied. The effects of various parameters, including taper parameter, profile, and grading function on the natural frequencies are investigated. Also, a series of finite element simulations are performed for comparison purposes. It is observed that the obtained results are in good agreement with the numerical simulations and available published data with vastly reduced computational cost as a result of using the collocation method.
\end{abstract}

